\title{
SISTEMAS ELECTORALES Y ESTILOS DE CAMPAÑA: LOS DIPUTADOS PANAMEÑOS Y EL VOTO PERSONALISTA*
}

\author{
Carlos Guevara Mann \\ Universidad de NeVADA-Reno, Estados Unidos
}

\begin{abstract}
Resumen
El método de elección de los diputados panameños no es muy conocido en el ámbito académico y sus efectos han sido poco estudiados. Aun cuando la Constitución de Panamá establece "el principio de representación proporcional”, la profusión de circunscripciones uninominales, aunada al sistema de mayoría relativa que se emplea para la distribución de escaños residuales en los circuitos plurinominales, hacen de dicho método, en la práctica, un sistema predominantemente mayoritario. En conjunto con lo anterior, la modalidad de lista abierta utilizada en las primeras dos fases de la asignación de escaños en circunscripciones plurinominales contribuye a promover el voto personalista sobre el voto partidario, ideológico o programático. Por tanto, como lo arguye este trabajo, los efectos del sistema electoral panameño coinciden con las predicciones teóricas sobre las consecuencias de los sistemas electorales en las campañas para diputado.
\end{abstract}

Abstract

The method for electing Panamanian deputies is not well known in academic circles and its effects have been insufficiently studied. The Panamanian Constitution establishes "the principle of proportional representation". In the distribution of remainders in multi-member districts, have given the system a predominantly majoritarian character. In conjunction with these features, the open-list system utilized in the first two allocation stages in multi-member districts contributes to promote the personal vote over partisan, ideological, or programmatic appeals. As argued in this paper, the effects of Panama's electoral system coincide with the theoretical predictions about the effects of electoral systems generally in congressional or parliamentary campaigns.

PALABRAS CLAVE • Panamá • Sistemas mayoritarios • Representación proporcional • Lista abierta $\bullet$ Voto personalista

\section{LOS SISTEMAS ELECTORALES Y SUS EFECTOS EN LAS CAMPAÑAS POLÍTICAS}

El sistema electoral es el método de acuerdo con el cual "Ios electores expresan su preferencia en votos" y dichos votos "se transforman en escaños" en la cámara representativa. Aunque existe una gran diversidad de sistemas electorales, esa vasta gama ha sido tradicionalmente agrupada en

Este artículo tiene su origen en una de las secciones de "Forsaken Virtue: An Analysis of the Political Behavior of Panamanian Legislators, 1984-1999", tesis de doctorado rendida en el Departamento de Gobierno y Relaciones Internacionales de la Universidad de Notre Dame en 2001. Una versión preliminar del artículo fue presentada en el Tercer Congreso Latinoamericano de Ciencias Políticas, celebrado en Campinas, Brasil, entre el 4 y 6 de septiembre de 2006. El autor agradece los comentarios de Gregory Schmidt, Juan Antonio Tejada Mora, David Altman y un revisor anónimo de la Revista de Ciencia Política, así como el apoyo financiero de la Universidad de Nevada, Reno, a través de su Programa de Apoyo a Actividades Académicas y Creativas (Scholarly and Creative Activities Grant Program). 
dos categorías principales: el sistema mayoritario y la representación proporcional (Antinori, 1995: 674-75; Nohlen, 2006) ${ }^{1}$. Los sistemas mayoritarios utilizan circunscripciones "uninominales" (0 sea, aquellas donde se elige un solo diputado) y emplean la fórmula mayoritaria para determinar al ganador: quien recibe una mayoría (absoluta o relativa) de votos gana el escaño.

La representación proporcional se basa en circunscripciones "plurinominales" (o sea, aquellas donde se elige a más de un diputado) y, generalmente, emplea listas donde están indicados los nombres de los candidatos. Estas listas pueden ser "abiertas" o "cerradas". Las listas abiertas (en inglés, "open lists") permiten a los electores votar por la nómina entera presentada por un partido, como también seleccionar al candidato o candidatos de su predilección y descartar a los otros integrantes de la lista. Las listas cerradas, por su parte, no permiten ese sufragio "preferencial" o "selectivo", sino únicamente el voto por la lista completa.

El propósito de la representación proporcional es reducir la disparidad entre la proporción de los sufragios que gana un partido a nivel nacional y el número de escaños que esa misma agrupación obtiene en la cámara representativa. A manera de ejemplo, si un partido recibe el $40 \%$ del voto para diputados, de acuerdo con el principio de representación proporcional debe obtener el $40 \%$ de los escaños en la asamblea (Reynolds y Reilly, 1997: 17-19).

Varios autores han examinado los efectos de los sistemas electorales en las candidaturas para diputado. En su distinción entre campañas en sistemas mayoritarios y de representación proporcional, Richard Katz sostiene que esta última generalmente promueve campañas ideológicas o programáticas, mientras que los sistemas mayoritarios estimulan candidaturas personalistas y localistas. Katz (1980: 28) explica el razonamiento detrás de esta predicción de la siguiente manera:

In PR [proportional representation] systems, voters choose parties rather than individuals. Because candidates are obliged to say "Vote for my party" rather than "Vote for me", it is more difficult for candidates of the same party to take different political lines. The importance of the corporate identity of the party is increased, and particularly the importance of the sense that its issue stands represent a unified program rather than a number of isolated proposals of individual candidates. Correspondingly, the general organizing principles underlying a specific proposal, that is the ideology, become more important.

Conversely, plurality electoral schemes encourage personalistic or localistic campaigning. Since the choice of voters is for candidates rather than for parties, candidates may attempt to minimize or even ignore their party affiliation, and the commitment to a specific platform that this implies. This naturally increases the relative importance of personality. Because local candidates are encouraged, localistic consideration will be of particular importance to some candidates (facing a local party challenge) and of some importance to most candidates (hoping to prevent such a challenge in the future) ${ }^{2}$.

1 En el artículo citado, Nohlen (2006) explica que "ya no basta con poder diferenciar entre sistemas de mayoría y representación proporcional. Y entre los sistemas combinados, por ejemplo, tampoco basta diferenciar entre sólo dos". Este punto de vista se asemeja al de Reynolds y Reilly (1997), quienes dividen los sistemas electorales en mayoritario, mixto y proporcional. No obstante ambas autorizadas opiniones, para evitar divagaciones que no son pertinentes a este trabajo se dispuso utilizar la tipología tradicional de los sistemas electorales.

2 "En los sistemas de representación proporcional, los votantes eligen partidos en vez de individuos. Debido a que los candidatos tienen la obligación de decir, "Vote por mi partido" en vez de "Vote por mí", es más difícil para 
Katz y otros autores también observan las diferencias entre estilos de campaña que se generan a partir del tipo de representación proporcional empleado en la distribución de escaños. En los sistemas de lista cerrada, los electores sólo pueden emitir su voto por el partido, sin expresar preferencia alguna al respecto de los candidatos listados en la papeleta. En otras palabras, es la dirigencia del partido la que determina quién es el individuo que ejerce la diputación. Por lo general, esa facultad discrecional debe ejercerse antes de que ocurra la elección. El partido normalmente presenta una lista de candidatos, entendiéndose que la proporción del voto que alcance el partido será asignada a los candidatos en el orden en que sus nombres aparecen en la lista (Katz, 1980: 31). Por cuanto los votantes emiten su voto por un partido, no por un individuo, la expectativa es que los candidatos en sistemas de lista cerrada promoverán en sus campañas la ideología y los programas de su partido.

Los sistemas de lista abierta, como el que se utiliza en los circuitos plurinominales panameños, permiten al votante expresar sus preferencias individuales entre varios candidatos de una lista partidaria (Katz, 1980: 31). Carey y Shugart (1995) coinciden en que uno de los efectos de la lista abierta es promover candidaturas basadas en reputaciones personales, o sea, la condición de ser muy conocido personalmente y estimado por los electores. Este punto de vista es plenamente compartido por Antinori (1995: 717-21). La tabla 1, presentada a continuación, resume estas predicciones teóricas sobre los efectos del sistema electoral en las campañas políticas:

TABLA 1: Predicciones teóricas sobre los efectos del sistema electoral (resumen)

\begin{tabular}{lcc}
\hline & Voto Personalista & $\begin{array}{c}\text { Voto Ideológico } \\
\text { por Partido }\end{array}$ \\
\hline Sistema mayoritario & $\mathrm{X}$ & \\
Representación proporcional-lista abierta & $\mathrm{X}$ & $\mathrm{X}$ \\
Representación proporcional-lista cerrada & & \\
\hline
\end{tabular}

Carey y Shugart sostienen que la popularidad personal constituye un valioso recurso de campaña en los lugares en que los candidatos compiten no solamente en contra de candidatos de otros partidos, sino además con copartidarios que aparecen en la misma lista, como en

los candidatos del mismo partido asumir líneas políticas distintas. La identidad corporativa del partido crece en importancia como aumenta también, particularmente, la percepción de que las posiciones programáticas representan un programa unificado en vez de un número de propuestas aisladas de candidatos individuales. De la misma manera, los principios organizativos generales que sustentan una propuesta específica -esto es, la ideología- adquieren mayor relevancia.

Los esquemas mayoritarios, contrariamente, promueven las campañas personalistas o localistas. Dado que los electores votan por candidatos en vez de partidos, los candidatos pueden tratar de minimizar o hasta obviar su afiliación partidaria y el compromiso con el conjunto de principios que dicha afiliación implica. Esta tendencia, naturalmente, aumenta la importancia relativa de la personalidad del candidato. El sistema mayoritario alienta las candidaturas locales, por lo que las consideraciones localistas tendrán particular importancia para algunos candidatos (los que enfrentan retos dentro de su propio partido en sus localidades) y de alguna importancia para la mayoría de los candidatos (que esperan prevenir dichos retos en el futuro)" (traducción del autor). 
Panamá. De acuerdo con este pronóstico, las candidaturas para diputado en Panamá deben tener un carácter esencialmente personalista. Como lo veremos más adelante, este vaticinio es acertado.

\section{UN SISTEMA HÍBRIDO}

El sistema de elección de los diputados panameños no es muy conocido en el ámbito académico y sus efectos han sido poco estudiados. Algunas de sus particularidades lo hacen merecedor de atención, especialmente en el marco del debate continuo sobre la conveniencia de los sistemas electorales que, como lo indica Nohlen (2006), es característico de las sociedades democráticas ${ }^{3}$. La Constitución panameña de 1972 (reformada en varias ocasiones, últimamente en 2004), garantiza "el principio de representación proporcional"; la elección de diputados en circuitos uninominales y plurinominales (ver Art. 147, numeral 1) ${ }^{4}$. Esa dualidad -la coexistencia, por disposición constitucional, de circuitos que emplean la fórmula mayoritaria con circunscripciones que utilizan la representación proporcional- produce una tensión conceptual que, en la práctica, se traduce en la disminución de la proporcionalidad del método de elección de los diputados panameños. La fórmula utilizada para la distribución de escaños residuales, tras la aplicación de las cuotas electorales en circuitos plurinominales, contribuye, aún más, a reducir la proporcionalidad del sistema. Como resultado, la mayoría de los diputados a la Asamblea Nacional son elegidos no de acuerdo con el sistema de representación proporcional, que consagra la Constitución, sino según un sistema de mayoría relativa (ver tabla 4).

En síntesis, a pesar de la garantía constitucional sobre "el principio de representación proporcional", el sistema electoral panameño es un sistema híbrido -literalmente, "producto de elementos de distinta naturaleza" ${ }^{-}$en el que predomina el método de elección por mayoría relativa. Como se verá más adelante, las disposiciones electorales panameñas concurren a la configuración de un estilo de campaña y, más generalmente, un estilo político que privilegia el personalismo. En el ámbito panameño, el personalismo, a su vez, promueve el clientelismo ${ }^{6}$, una práctica que reduce la calidad de la democracia y, a la larga, puede llevar a deslegitimar el régimen democrático. Como bien lo indica Nohlen (2006), un fenómeno tan complejo como el clientelismo no puede atribuirse exclusivamente al sistema electoral. Sin embargo, a partir de la premisa institucionalista de que las instituciones, incluyendo las de tipo electoral, contribuyen a surtir efectos políticos

Sobre el particular, ver el expediente sobre la reforma del sistema binominal chileno, contenido en la Revista de Ciencia Política 26 (1): 191-235.

$4 \quad$ Las constituciones panameñas estaban disponibles el 5 de octubre de 2006 en: www.binal.ac.pa/buscar/clconst. htm. La disposición referida estatuye: “Habrá circuitos uninominales y plurinominales, garantizándose el principio de representación proporcional".

5 Esta es una de las acepciones asignadas a la palabra "híbrido" en el Diccionario de la Lengua Española. Ver la versión electrónica del diccionario en http: //www.rae.es, s.v. "híbrido".

6 Entiéndese por clientelismo "la asignación de bienes públicos como si fueran favores personales a clientelas políticas, a cambio de apoyo para el jefe político. Criterios políticos (tales como adhesión a una persona o partido) y conexiones personales prevalecen sobre otras posibles formas de selección de beneficiarios de programas estatales, tales como el derecho universal al acceso a dichos programas, la selección aleatoria, la meritocracia o la eficiencia (Mainwaring, 1999: 178, traducción del autor). 
(Putnam, 1993), de las predicciones teóricas sobre las consecuencias de las instituciones electorales resumidas en la sección anterior y de la evidencia empírica recabada en varios regímenes democráticos, especialmente los de mayor longevidad, tampoco cabe duda de que el sistema electoral ayuda a moldear la conducta de los políticos.

\section{EL DISEÑO ELECTORAL DE LA ASAMBLEA NACIONAL}

Ilustrar la conexión entre formulas electorales y campañas personalistas en Panamá requiere, en primer lugar, un examen del diseño electoral de la Asamblea. El sistema electoral panameño está estipulado en la Constitución de 1972, reformada en 1978, 1983, 1994 y 2004, y el Código Electoral, cuya actualización más reciente data de 2002 (República de Panamá, 1994, 2003, 2004). La revisión del Código Electoral para armonizar su contenido con la reforma constitucional de 2004 todavía está pendiente. La Comisión Nacional de Reforma Electoral presentó un texto modificado en febrero de 2006 (Tribunal Electoral, IV Comisión, 2006). A la fecha en que fue redactado este artículo, dicho texto aún no había sido considerado en primer debate por la Comisión de Gobierno, Justicia y Asuntos Constitucionales de la cámara (Sánchez, 2006) ${ }^{7}$.

La Asamblea Nacional de Panamá es una corporación unicameral constituida, en la actualidad, por 78 diputados elegidos en 41 circuitos electorales por un período de cinco años ${ }^{8}$. De estas 41 circunscripciones, 27 son uninominales y 14 son plurinominales. En los circuitos plurinominales se eligieron en 2004 entre dos y ocho diputados. A cada circunscripción electoral corresponde un número compuesto de dos cifras, la primera de las cuales representa la provincia en la que está localizado el circuito. Los circuitos plurinominales más grandes (cuyo número comienza con 8 en representación de la Provincia de Panamá) están localizados en la capital o sus municipios aledaños, que forman parte del área urbana de la zona de tránsito (Antinori, 1995: 543). La tabla 2 enumera, a continuación, los circuitos plurinominales que sirvieron de base para la elección de diputados en 2004 y el número de diputados (o magnitud de circuito [M]) de cada circunscripción: 
TABLA 2: Circuitos electorales y magnitud de circuito (M) (Panamá, 2004)

\begin{tabular}{clc}
\hline Circuito & Localización & M \\
\hline 8.10 & Parte del Distrito de Panamá (área urbana de la zona de tránsito) & 8 \\
8.7 & San Miguelito (área urbana de la zona de tránsito) & 7 \\
8.9 & Parte del Distrito de Panamá (área urbana de la zona de tránsito) & 5 \\
8.8 & Parte del Distrito de Panamá (área urbana de la zona de tránsito) & 4 \\
8.1 & Arraiján (área urbana de la zona de tránsito) & 4 \\
3.1 & Colón (área urbana de la zona de tránsito) & 4 \\
8.7 & Parte del Distrito de Panamá (área urbana de la zona de tránsito) & 3 \\
8.5 & La Chorrera (área urbana de la zona de tránsito) & 3 \\
4.1 & David (Provincia de Chiriquí) & 3 \\
9.1 & Santiago (Provincia de Veraguas) & 2 \\
2.1 & Penonomé (Provincia de Coclé) & 2 \\
1.1 & Bocas del Toro (Provincia de Bocas del Toro) & 2 \\
4.2 & Barú (Provincia de Chiriquí) & 2 \\
4.3 & Bugaba (Provincia de Chiriquí) & 2 \\
Uninominales & 27 circuitos, principalmente rurales & 1 \\
\hline
\end{tabular}

Fuente: Tribunal Electoral, 2004b.

Los integrantes de la Asamblea son elegidos al mismo tiempo que todos los otros cargos de elección, incluyendo al presidente y vicepresidente de la República, 20 diputados al Parlamento Centroamericano, los alcaldes municipales (que en 2004 fueron 75) y los representantes de corregimiento (que en 2004 fueron 619). Para suplir vacantes en la Asamblea, se eligió a dos suplentes por cada diputado principal hasta 2004. La reforma constitucional de 2004 eliminó al segundo suplente. La más reciente elección general fue celebrada en mayo de 2004. La próxima elección general deberá celebrarse en mayo de 2009.

Los diputados actuales fueron elegidos de acuerdo con el artículo 141 de la Constitución de 1972, vigente en mayo de 2004. Posteriormente, la reforma constitucional de 2004 modificó los principios de configuración de los circuitos electorales. Las nuevas normas, que fijan el número de diputados en 71 y, en consecuencia, requieren la reconfiguración de las circunscripciones electorales, tendrán vigencia en las elecciones de 2009.

Antes de la reforma constitucional de 2004, para la elección de diputados el país estaba dividido, tal como fue expuesto, en 41 circuitos electorales. El artículo 141 de la Constitución de 1972, vigente hasta 2004, establecía los parámetros básicos de la división electoral de acuerdo con una metodología complicada, cuyos términos literales eran los siguientes:

Artículo 141. La Asamblea Legislativa [Nacional] se compondrá de los Legisladores [Diputados] ${ }^{9}$ que resulten elegidos en cada Circuito Electoral, de conformidad con las bases siguientes: 
1. Cada Provincia y la Comarca de San Blas se dividirán en Circuitos Electorales.

2. La Provincia de Darién y la Comarca de San Blas tendrán dos Circuitos Electorales cada una y en éstos se elegirá un Legislador por cada Circuito Electoral.

3. Los actuales Distritos Administrativos que, según el último Censo Nacional de Población, excedan de cuarenta mil habitantes, formarán un Circuito Electoral cada uno y en tales circuitos se elegirá un Legislador por cada treinta mil habitantes y uno más por residuo que no baje de diez mil. El Distrito de Panamá se dividirá a su vez en cuatro Circuitos Electorales, de conformidad con el numeral cinco de este artículo y según lo disponga la Ley. En los Circuitos Electorales en que se deben elegir a dos o más Legisladores, la elección se hará conforme al sistema de representación proporcional que establezca la Ley.

4. Excepto la Provincia de Darién, la Comarca de San Blas y los Distritos Administrativos actuales a que se refiere el numeral tres, anterior, en cada Provincia habrá tantos Circuitos Electorales cuantos correspondan a razón de uno por cada treinta mil habitantes y uno más por residuo que no baje de diez mil, según el último Censo Nacional de Población, previa deducción de la población que corresponde a los actuales Distritos Administrativos de que trata el numeral tres. En cada uno de dichos Circuitos Electorales se elegirá un Legislador.

5. Cada Circuito Electoral tendrá un máximo de cuarenta mil habitantes y un mínimo de veinte mil habitantes, pero la Ley podrá crear Circuitos Electorales que excedan el máximo o reduzcan el mínimo anteriores, para tomar en cuenta las divisiones políticas actuales, la proximidad territorial, la concentración de la población indígena, los lazos de vecindad, las vías de comunicación y los factores históricos y culturales, como criterios básicos para el agrupamiento de la población en Circuitos Electorales (República de Panamá, 1994).

La aplicación del artículo 141 a la práctica significó una división electoral del país en una mayoría de circuitos uninominales (27 en 2004) y una minoría de circunscripciones plurinominales (14 en 2004) de baja magnitud ${ }^{10}$, lo cual, como se mencionó anteriormente, presenta, en la práctica, una contradicción con la garantía de representación proporcional contenida en la Constitución. En los circuitos uninominales se emplea la fórmula mayoritaria para identificar a los candidatos vencedores. En esos circuitos, el candidato que obtiene el voto mayoritario es proclamado vencedor. No se requiere una mayoría absoluta, sino relativa, para ganar un escaño en los circuitos uninominales (ver el 291 del Código Electoral vigente en República de Panamá, 2003).

\section{EL MÉTODO DE ASIGNACIÓN DE ESCAÑOS EN CIRCUITOS PLURINOMINALES}

En los circuitos plurinominales, los diputados son elegidos mediante listas abiertas (voto preferencial o selectivo), según lo señala el artículo 292 del Código Electoral vigente. Hay tres etapas para la asignación de escaños. En la primera se emplea el cociente electoral simple. En

10 Para un análisis de los efectos del reducido tamaño de las circunscripciones electorales sobre la calidad de la representación política en Panamá, ver Guevara Mann, 2004. 
la segunda, se aplica la mitad del cociente (o "medio cociente"). Los asientos que queden sin asignar tras la aplicación del cociente y el medio cociente se distribuyen, en la tercera fase, a los candidatos (no a los partidos) que hayan recibido el mayor número de votos preferenciales (exceptuando, obviamente, a los candidatos a los que se les fueron adjudicados escaños mediante cociente o medio cociente). El artículo 292 del Código Electoral ordena este procedimiento en los siguientes términos:

Cuando se trate de circuitos electorales que elijan a dos o más Legisladores [Diputados], las Juntas de Escrutinio de Circuito Electoral proclamarán a los candidatos electos de conformidad con las siguientes reglas:

1. El número total de votos válidos depositados en el Circuito por todos los electores se dividirá por el número de ciudadanos que han de elegirse. El resultado de esta división se denominará cuociente [sic] electoral.

2. El número total de papeletas obtenidas por cada lista de candidatos se dividirá por el cuociente electoral y el resultado de esta operación será el número de candidatos que corresponde elegir al partido que hubiere lanzado la lista de que se trata.

3. Si quedasen puestos por llenar para completar el número de ciudadanos que han de elegirse, se adjudicará uno a cada una de las listas restantes que hayan obtenido un número de papeletas no menor de la mitad del cuociente electoral en el orden en que dichas listas hayan obtenido papeletas. Los partidos que hayan obtenido el cuociente electoral no tendrán derecho al medio cuociente.

4. Si aún quedaran puestos por llenar, se adjudicarán a los candidatos más votados, una vez aplicado el cuociente y medio cuociente. Para la adjudicación del puesto por residuo, se contarán todos los votos obtenidos por cada candidato en todas las listas en que hayan sido postulados. Pero, en todo caso, la curul se asignará al partido que le haya aportado la mayor cantidad de votos al candidato.

Si ningún partido alcanzare cuociente ni medio cuociente, serán elegidos los candidatos que más votos hayan obtenido, sumándose los votos que hayan obtenido en todas las boletas 0 listas de candidatos en que aparecieren (República de Panamá, 2003, énfasis añadido).

La fórmula tripartita de elección de los diputados panameños se remonta a 1925, cuando se la introdujo en la legislación panameña. Aunque la distribución por cociente tiene evidentes similitudes con el método propuesto por Thomas Hare (la llamada "cuota de Hare"), de acuerdo con el Profesor César Quintero (1967), prominente consitucionalista panameño, el sistema electoral panameño, del cociente, medio cociente y residuo, fue esbozado por Nikolaus Saripolos, quien lo expuso en La Démocratie et l'election proportionelle (La democracia y la elección proporcional), obra publicada en París, en 1899. Con algunas oscilaciones -tales como la eliminación del medio cociente y variaciones en la regla para la distribución de residuos- el sistema de cocientes se aplicó en Panamá desde las elecciones de 1928 hasta el golpe militar de 1968 (Antinori, 1995: 731, 733). Fue reintroducido en 1984, cuando el régimen militar permitió la celebración de elecciones (muy manipuladas) a una "Asamblea Legislativa" ostensiblemente diseñada de acuerdo con principios democráticos, como resultado de la reforma constitucional de 1983. 
En este punto conviene ilustrar, con un ejemplo práctico, la operación del método para la distribución de escaños en los circuitos plurinominales panameños. En la primera etapa, las diputaciones se asignan a los partidos que obtienen un número de votos equivalente al cociente electoral. En otras palabras, si en un circuito que elige cinco diputados se emiten 100.000 votos válidos, cada partido que recibe por lo menos la cuota completa de 20.000 votos $(100.000 \div 5)$ recibe un puesto en la Asamblea Nacional.

En la segunda etapa, los escaños sin asignar se distribuyen a los partidos que obtienen un número de votos equivalente a la mitad del cociente electoral (i.e., 10.000 votos en el ejemplo señalado). De acuerdo con el Código Electoral vigente, los partidos que obtienen el cociente electoral no tienen derecho a la asignación de escaños por medio cociente.

En cada una de estas dos etapas, la identidad de los candidatos vencedores se determina con base en los votos preferenciales o selectivos recibidos por cada candidato en las listas partidarias, de acuerdo con lo que dispone el artículo 293 del Código Electoral (República de Panamá, 2003). Además de emitir su voto por una de las listas, cada elector tiene tantos votos preferenciales cuantos candidatos haya en la papeleta de su predilección. Los electores no tienen la obligación de ejercer su voto selectivo (en cuyo caso se contabiliza un voto preferencial a cada uno de los integrantes de la lista). Si deciden ejercer su voto selectivo, deben marcar la casilla anexa al nombre del candidato o los candidatos de su preferencia.

Los candidatos suelen pedirles a sus seguidores que expresen sus preferencias por ellos. De esta manera, buscan maximizar sus posibilidades de ser elegidos diputados. Y para persuadir a los electores de la conveniencia de utilizar su voto preferencial, los candidatos implementan estrategias de campaña que destacan su imagen como proveedores de bienes públicos y servicios personales. Como lo anticipa la teoría, el sistema de lista abierta promueve, en Panamá, candidaturas personalistas y no ideológicas (Antinori, 1995: 717-21). A ese personalismo contribuye, además, la fórmula para la asignación de escaños residuales.

\section{LA REGLA DEL RESIDUO Y SU EVOLUCIÓN}

Como se indicó anteriormente, en la tercera etapa de la adjudicación de diputaciones los escaños que quedan por distribuir en circuitos plurinominales se asignan mediante una metodología mayoritaria, que se aleja del sistema de representación proporcional. Desde 1983, el método para la asignación de residuos ha evolucionado como sigue. En las elecciones de 1984 (bajo el régimen castrense) los escaños tras la distribución mediante cociente y medio cociente fueron asignados a los partidos (“listas restantes”) que obtuvieron los residuos más altos, tras deducir un cociente electoral a las agrupaciones que obtuvieron dicho cociente y medio cociente electoral a los partidos que obtuvieron dicho medio cociente. Al menos en teoría, este procedimiento permitía una distribución más amplia entre los partidos que participaban en la elección. En la práctica, sin embargo, una combinación de clientelismo, tácticas intimidatorias y fraude electoral produjo, en la Asamblea Legislativa, una mayoría constituida por el PRD, el partido del régimen militar y sus satélites ${ }^{11}$. 
En 1989, los escaños residuales fueron asignados a los partidos con los remanentes más altos, después de restarles medio cociente a los partidos que recibieron curules por cociente entero o medio cociente. La distribución del residuo tuvo lugar sólo entre los partidos que recibieron escaños por cociente o medio cociente. Aunque estas elecciones fueron celebradas bajo el régimen militar, la adjudicación de diputaciones no tuvo lugar hasta después del desalojo de la dictadura (mediante una acción armada estadounidense) y la restauración del régimen civil en diciembre de 1989.

Por iniciativa de la fracción mayoritaria del Partido Demócrata Cristiano, en 1993 la Asamblea aprobó una modificación al Código Electoral mediante la cual los escaños residuales serían asignados a los candidatos (no a los partidos) que recibieran la mayor cantidad de votos selectivos. Tras su aprobación, este método fue utilizado en las elecciones de 1994, 1999 y 2004. Como resulta evidente, las reformas electorales adoptadas en Panamá desde la década de 1980 han mermado la proporcionalidad de la fórmula de distribución de escaños en circuitos plurinominales.

La regla del residuo aplicada desde la reforma electoral de 1993 requiere la adjudicación de bancas residuales a los candidatos que reciban la mayor cantidad de votos selectivos en cada circuito, independientemente de la proporción del voto o el número de sufragios recibidos por cada partido por cociente o medio cociente (Valdés Escoffery, 2002). En otras palabras, en la asignación de residuos no se descuentan los votos utilizados para asignar escaños por cociente o medio cociente a los partidos. Estos votos son contabilizados dos veces, como votos por partido y votos preferenciales. En el ejemplo antes utilizado, si un partido obtiene 24.000 votos y recibe, por lo tanto, un escaño por cociente, la cuota completa de 20.000 votos no se descuenta a los candidatos de ese partido para la adjudicación del residuo. En consecuencia, el candidato de la lista que reciba el voto selectivo más alto y que no haya sido proclamado ganador por cociente o medio cociente contabiliza la cuota de 20.000 votos dentro de su voto preferencial, el cual se computa como 24.000 en vez de 4.000 sufragios. Esta fórmula, que tergiversa los resultados electorales en favor de los partidos más grandes, ha generado quejas y un reclamo ante la Comisión Interamericana de Derechos Humanos (Morice, 2000). La tabla 3 resume el sistema de reparto de escaños empleado en los circuitos plurinominales panameños en 1994, 1999 y 2004.

En la tercera etapa del proceso de distribución, el método de asignación de escaños utilizado en 1994, 1999 y 2004 es una fórmula mayoritaria. Como resultado de una reducida magnitud de distrito $(\mathrm{M})$-caracterizada por una prevalencia de circuitos uninominales- y una regla para la distribución de residuos fundamentada en una lógica mayoritaria, la mayoría de los diputados panameños es elegida con base en un sistema mayoritario, no proporcional. La tabla 4 muestra el número de escaños asignados por mayoría relativa y el método proporcional en las elecciones de 2004. Como lo demuestra dicha tabla, en los comicios más recientes, 41 de los 78 puestos de la cámara (53\%) fueron asignados por mayoría relativa. 
TABLA 3: Sistema de distribución de escaños en circuitos plurinominales (Panamá, 1994, 1999 y 2004)

\section{Etapa de}

Asignación

Cociente entero

Medio cociente

Residuo

\section{Método}

Los escaños se asignan a los partidos que reciben un número de votos equivalente a la cuota electoral completa, i.e., el total de votos válidos emitidos en una circunscripción dividido entre el número de diputados por elegir en el circuito. Se proclaman como ganadores a los candidatos que reciben el mayor número de votos preferenciales en cada lista a la que corresponda una cuota electoral completa ("cuociente entero").

Los escaños se asignan a los partidos que reciben un número de votos equivalente a la mitad de la cuota electoral, i.e., el total de votos válidos emitidos en una circunscripción dividido entre el número de diputados por elegir en el circuito, dividido entre dos. Los partidos que obtuvieron escaños por cociente electoral no participan en la distribución por medio cociente. Se proclaman como ganadores a los candidatos que reciben el mayor número de votos preferenciales en cada lista a la que corresponda una cuota electoral completa ("medio cociente").

Los escaños se asignan a los candidatos (no a los partidos) que reciben el mayor número de votos preferenciales o selectivos en cada lista, que no hayan sido proclamados mediante la metodología del cociente entero o medio cociente.

Fuente: Artículos 292 y 293 del Código Electoral en República de Panamá, 2003.

TABLA 4: Fórmulas para la asignación de diputaciones y número de escaños asignados de acuerdo con cada fórmula (Panamá, 2004)

\begin{tabular}{clrrrrrr}
\hline & \multirow{2}{*}{ Fórmula } & \multicolumn{4}{c}{ Número y Porcentaje de Escaños } \\
\cline { 3 - 8 } & & \multicolumn{2}{c}{1994} & \multicolumn{2}{c}{1999} & \multicolumn{2}{c}{2004} \\
\cline { 2 - 8 } & Cociente entero & $\mathrm{N}$ & $\%$ & $\mathrm{~N}$ & $\%$ & $\mathrm{~N}$ & \multicolumn{1}{c}{$\%$} \\
\hline \multirow{2}{*}{$\begin{array}{c}\text { Representación } \\
\text { Proporcional }\end{array}$} & Medio cociente & 20 & $28 \%$ & 11 & $15 \%$ & 17 & $22 \%$ \\
& Subtotal & 26 & $36 \%$ & 29 & $40 \%$ & 37 & $47 \%$ \\
\hline \multirow{2}{*}{ Mayoría } & Residuo (plurinominales) & 19 & $27 \%$ & 16 & $23 \%$ & 14 & $18 \%$ \\
relativa & Uninominales & 26 & $36 \%$ & 26 & $37 \%$ & 27 & $35 \%$ \\
& Subtotal & 45 & $63 \%$ & 42 & $60 \%$ & 41 & $53 \%$ \\
\hline Art. 14112 & Partidos sin representación & 1 & $1 \%$ & 0 & $0 \%$ & 0 & $0 \%$ \\
\hline \multirow{2}{*}{} & N total & 72 & $100 \%$ & 71 & $100 \%$ & 78 & $100 \%$ \\
\hline
\end{tabular}

Fuentes: Tribunal Electoral, 1999a, 1999b, 2004a.

12 La adjudicación de escaños contenida en el Art. 141, numeral 6 de la Constitución de 1972, anterior a su reforma de 2004, consistía en lo siguiente: “Los partidos políticos que hubieren alcanzado el número de votos exigidos para subsistir como tales, y que no hayan logrado la elección de un Legislador en algún Circuito Electoral, tienen derecho a que se les adjudique un escaño de Legislador. La adjudicación se hará en favor del candidato que hubiere obtenido mayor número de votos para Legislador, dentro de su partido". 
La reforma electoral presentada a la Asamblea Nacional en marzo de 2006 (y aún pendiente de consideración por la cámara) propone un retorno a la fórmula de 1989, mediante la cual se resta medio cociente a los partidos que reciben escaños por cociente entero o medio cociente. Sólo los partidos que reciban diputaciones por cociente o medio cociente participarían en la asignación de residuos ${ }^{13}$. De ser aprobadas, ambas disposiciones mantendrían vigente la tendencia a favorecer a los partidos más grandes, en detrimento de la proporcionalidad del sistema electoral.

\section{LAS CAMPAÑAS DE LOS DIPUTADOS Y EL VOTO PERSONALISTA ${ }^{14}$}

De acuerdo con la teoría sobre los efectos de los sistemas de elección de diputados, brevemente esbozada al inicio del artículo, dependiendo del régimen electoral imperante en un país las campañas políticas pueden tener contenido principalmente personalista o ideológico. En sistemas de representación proporcional con lista cerrada deben predominar las campañas políticas de corte ideológico, que destaquen la base programática del partido nominador. En sistemas mayoritarios o de representación proporcional con lista abierta, tienden a prevalecer las campañas políticas de corte personalista. Esta teoría predice correctamente el carácter de las campañas políticas para diputado en Panamá. Para corroborar dicha predicción, ésta y las siguientes secciones ofrecen un vistazo general a la dinámica electoral en Panamá. Un análisis más sistemático y detallado, por emprenderse en otro contexto, suministrará conclusiones más específicas.

En sistemas electorales como el de Panamá, en el que más de la mitad de los integrantes de la Asamblea es elegida de acuerdo con fórmulas mayoritarias, los candidatos destacan sus logros personales más que el contenido ideológico de los partidos a los que pertenecen (que no suele ser ni muy sólido ni muy profundo). Esto no significa que las campañas carecen por completo de referencias al partido. El partido es, después de todo, la entidad que presenta candidatos; hasta la reforma electoral de 2004, correspondía exclusivamente a los partidos la nominación de candidatos a diputado ${ }^{15}$. Sin embargo, esa referencia por lo general se limita a relacionar al candidato con el nombre, los colores y los símbolos del partido que lo presenta, así como el número que le corresponde en la lista partidaria (Antinori, 1995: 720).

Más que la ideología del partido al que pertenece (o que lo presenta), en la carta de presentación del candidato figuran, prominentemente, sus logros personales. En el sistema electoral panameño, los candidatos que pueden hacer gala de logros individuales significativos tienen mayores posibilidades de recibir los votos selectivos necesarios para asegurar su elección a un escaño

13 Ver la propuesta de modificación en Tribunal Electoral, IV Comisión, 2004.

14 Esta sección está basada en Guevara Mann, 2001; Antinori, 1995; observaciones personales del autor durante las campañas políticas de 1984, 1989, 1994, 1999 y 2004, y entrevistas efectuadas en 2006 al ex candidato presidencial José Miguel Alemán, los ex diputados Jorge Rubén Rosas y Carlos Iván Zúñiga, el ex magistrado suplente de la Corte Suprema de Justicia, Juan Antonio Tejada Mora, y el ex director de La Prensa, Juan Arias Zubieta.

15 La reforma constitucional de 2004, aprobada luego de las elecciones generales de ese año, introdujo la llamada “libre postulación” de candidatos a la Asamblea Nacional. Entre 1984 y 2004, sólo los partidos políticos podían presentar candidatos a la Asamblea. Ver, sobre el particular, el artículo 146 de la Constitución de 1972, tras la reforma de 2004 y comparar con el artículo 140 de la misma Constitución, antes de la reforma de 2004, en www. binal.ac.pa/buscar/clconst.htm. 
que quienes no son capaces de presentar antecedentes creíbles como proveedores de servicios comunitarios o personales. Aunque es aventurado afirmar que el método electoral explica completamente la naturaleza de la cultura política del país, sí puede decirse que contribuye a entender el carácter personalista y poco programático de la política electoral panameña.

En su ya clásica obra sobre el comportamiento político de los congresistas estadounidenses, Mayhew (1974) identifica tres actividades fundamentales en que incurren los diputados a fin de conseguir su reelección: la promoción o publicidad (advertising), la atribución de crédito (credit claiming) y la toma de posiciones (position-taking). Esta tipología de actividades de campaña es útil para aproximarnos, con mayor precisión, a la naturaleza de las campañas políticas en Panamá.

La lógica principal que impulsa las campañas a la Asamblea panameña es que los candidatos necesitan maximizar su visibilidad y el reconocimiento de sus nombres para lograr la elección a la cámara (Alemán, 2006). Con ese propósito llevan a cabo, principalmente, actividades de promoción o publicidad y atribución de crédito. La tercera actividad destacada por Mayhew -la toma de posiciones- no es tan relevante, evidentemente por el énfasis personalista de las campañas políticas. Esta última actividad tiene un carácter más ideológico que no es tan conducente al voto personalista como lo son las otras dos. Son pocos los candidatos a diputado que participan en debates públicos sobre temas nacionales, emiten programas políticos o documentos de compromiso con sus electores -y menos aún los que publican columnas de opinión en los diarios- acciones que pudiesen catalogarse bajo el rótulo de "toma de posiciones". Las actividades que con mayor prominencia figuran en las estrategias de campaña en Panamá son aquellas vinculadas con la promoción o publicidad y la atribución de crédito.

\section{LA PROMOCIÓN O PUBLICIDAD COMO ESTRATEGIA DE CAMPAÑA}

En Panamá, los aspirantes a una diputación promueven sus candidaturas profusamente. La más elemental de las actividades de promoción -que se considera esencial para el triunfo electoral-consiste en caminar (o conducir) por el circuito electoral para presentarse personalmente a los electores. Esta actividad va de la mano con acciones para atribuirse crédito.

Todos los candidatos "caminan" sus distritos, frecuentemente acompañados por sus familiares y colaboradores y, en ocasiones, por simpatizantes prominentes y bandas de músicos (llamadas “murgas" en Panamá). El objetivo principal de estas giras "circuitales" es permitirle al candidato revelarse como un individuo accesible, capaz de interactuar con el ciudadano común y corriente y de ayudarlo a resolver sus problemas personales y, por lo tanto, merecedor del voto preferencial o selectivo. En estas ocasiones, los electores presentan cientos de peticiones al candidato: de ayuda para conseguir un empleo o un subsidio gubernamental 0 , abiertamente, de dinero en efectivo para superar alguna dificultad personal. La mayoría de los candidatos lleva consigo algún material promocional para distribuir a los electores: gorros, camisetas, tazas, vasos, bolígrafos, lápices, calendarios, cuadernos, llaveros, paraguas, linternas, bolsas de comida y caramelos y otros artículos, todos impresos con el nombre del candidato, sus colores, símbolos y lemas (y los del partido), así como el número del aspirante en la lista electoral del partido. Durante estas giras, algunos candidatos patrocinan eventos deportivos y celebran sorteos en los que se rifan aparatos electrodomésticos, entre ellos ventiladores, radios, televisores, neveras, máquinas de 
lavar y estufas. Además de ayudarlos a darse a conocer en el circuito, los patrocinios y sorteos les permiten a los candidatos atribuirse crédito como individuos preocupados por el bienestar de los pobladores del circuito.

La distribución de hojas volantes y afiches, que se entregan a los votantes o se adhieren a las paredes, es otra actividad promocional de consideración. En las semanas próximas a los comicios, los espacios urbanos se llenan por completo con avisos de campaña que contienen grandes imágenes de cada candidato, con el propósito de aumentar su visibilidad y reconocimiento. El Código Electoral, que prohíbe la remoción de anuncios de campaña por terceras personas, sólo ampara a los árboles, las estructuras públicas y ciertas edificaciones (escuelas, hospitales, templos) contra la adhesión de carteles electorales ${ }^{16}$. Para promover sus candidaturas, muchos aspirantes a una diputación también colocan avisos en los periódicos o en emisoras de radio y televisión. Como la música es un componente importante de la cultura popular panameña, los candidatos que pueden permitírselo incluyen, en su publicidad radial o televisiva, canciones 0 tonadas contagiosas que los electores pueden asimilar y repetir.

Estas actividades son significativamente más costosas que la distribución de afiches. Como en otros países, en Panamá la publicidad radial y, sobre todo, televisiva es sumamente costosa (Alemán, 2006). En efecto, las televisoras y radiodifusoras aguardan el período electoral con gran expectativa, por el aumento significativo en sus ingresos que las campañas les producen ${ }^{17}$. Como los partidos generalmente no financian las campañas de sus candidatos a la Asamblea, éstos se ven obligados a conseguir patrocinadores, gestionar préstamos bancarios, llevar a cabo actividades de recaudación de fondos (cenas, rifas) o utilizar recursos personales o familiares para financiar sofisticadas estrategias de promoción que se consideran importantes para asegurar el éxito electoral (Alemán, 2006; Antinori, 1995: 718-19).

En 1994, una candidatura de alta visibilidad en uno de los circuitos más grandes costaba alrededor de US\$150.000. Diez años más tarde, el costo de una campaña similar para un candidato gubernamental, con acceso a los recursos del Estado, se situaba entre US $\$ 250.000$ y US $\$ 300.000$ (Alemán, 2006). Este fue el costo estimado de una campaña en una circunscripción cuyo cociente entero fue de 21.500 votos. Como promedio, entonces, cada voto en dicho circuito le costó a uno de los candidatos más populares (que logró la reelección) entre US $\$ 11,63$ y US $\$ 13,95$, fuera del apoyo gubernamental en obras y servicios que consiguió para su circunscripción. Estas estrategias comprometen a muchos candidatos con poderosos patrocinadores, un rasgo de la política panameña (y de otros países) que menoscaba su autonomía y, eventualmente, puede distorsionar el vínculo entre electores y representantes.

\section{LA ATRIBUCIÓN DE CRÉDITO Y LOS SERVICIOS PERSONALES}

En sus actividades promocionales, los candidatos a la Asamblea panameña suelen atribuirse crédito como proveedores de servicios personales y bienes públicos. Un desempeño exitoso

16 Ver el Art. 185 del Código Electoral en República de Panamá 2003.

17 Observación personal del autor en su condición de gerente de crédito de la sucursal panameña de uno de los principales bancos internacionales (2001-2003). 
como agente político en la distribución de bienes y servicios apetecidos por la población se considera necesario para solicitar el voto selectivo. En el modelo representativo imperante en Panamá, la prestación de servicios es el componente fundamental y así lo ha sido a lo largo de la historia, como lo indica un breve repaso de la carrera de los diputados con mayores años de labor en la Asamblea.

Desde la primera sesión de la Asamblea en 1904, más de 700 individuos han fungido como diputados, pero ninguno ha ganado más de cinco elecciones. Sólo cuatro personas alcanzaron un escaño en cinco ocasiones: Jacinto López y León, Pablo Othón, Juan B. Arias y Jorge Rubén Rosas $^{18}$. La carrera de los primeros tres se desarrolló en el periodo anterior a la dictadura militar; Jorge Rubén Rosas fue diputado antes y después del golpe militar de 1968 (por un partido de oposición al régimen castrense). En los cuatro casos, la aptitud para satisfacer las necesidades personales de muchos electores fue instrumental para conseguir los votos selectivos necesarios para lograr la elección a la Asamblea.

A través de organizaciones locales eficientes, frecuentemente compuestas por empleados públicos cuyos cargos eran gestionados por los propios diputados, estos miembros de la Asamblea ayudaban a sus seguidores a resolver dificultades económicas (desempleo, enfermedades, muerte de algún familiar y otras calamidades), solucionar problemas con el gobierno y acceder a oportunidades educativas o laborales. Gracias a su influencia con el gobierno central, los diputados conseguían, además, la asignación de partidas presupuestarias para la construcción de obras de infraestructura en sus comunidades, tales como escuelas, plantas eléctricas, acueductos, puentes, caminos y centros de salud, así como para equipar dichas instalaciones y nombrarles personal contratado en las localidades.

Aunque algunos de estos diputados varias veces reelegidos también adquirieron sólidas reputaciones como legisladores idóneos y competentes fiscalizadores de la administración pública -particularmente los diputados Arias, López y Rosas- no eran éstas las actividades que sustentaban la conexión con sus electores (Zúñiga, 2006). De hecho, la razón por la cual Rosas atribuye su única derrota electoral (frente a su suplente) en 1994 fue su incapacidad para atender las necesidades de su circuito durante el período 1989-1994. Entre 1989 y 1993, Rosas ejerció el cargo de Ministro de Trabajo y Bienestar Social en el gabinete del Presidente Guillermo Endara, para lo cual tuvo que pedir licencia de su diputación. Aunque el cargo ministerial le ganó reconocimiento nacional, el Ministerio de Trabajo no maneja los recursos necesarios para satisfacer las aspiraciones circuito rural que lo envió a la Asamblea (Rosas, 2006).

18 Con la excepción de Juan B. Arias, estos diputados no lo fueron durante períodos consecutivos. Jacinto López y León representó a la Provincia de Los Santos, una región rural, por primera vez en 1928-1932 y por última ocasión en 1960-1964. Pablo Othón fue elegido por primera vez en 1932, como diputado por la Provincia del Darién, un área remota colindante con Colombia. Othón culminó su labor representativa en 1960. En representación de la Provincia de Panamá, la circunscripción electoral que incluía entonces al principal centro urbano del país, Juan B. Arias ganó cinco elecciones consecutivas a partir de 1948. Jorge Rubén Rosas ganó tres elecciones consecutivas entre 1960 y 1968 (cuando el golpe militar clausuró la Asamblea Nacional) y dos elecciones posteriores, en 1984 y 1989. Al inicio, Rosas fue diputado por la Provincia de Chiriquí. Tras la introducción de un nuevo regimen electoral en 1983, Rosas representó en la Asamblea, como legislador de un partido opositor a la dictadura (Molirena), a un circuito electoral pequeño en la sección oriental de la misma Provincia. 
Si el servicio a los electores es clave para garantizar la ganancia electoral, los diputados alineados con el gobierno tienen ventajas sobre aspirantes que no lo son o, inclusive, sobre miembros de la oposición. Esta es una de las razones que contribuye a explicar no sólo el aumento en la tasa de reelección desde $1984^{19}$, sino además el éxito del gobierno en atraer a diputados de oposición a las huestes oficialistas. Los diputados que regularmente colaboran con el gobierno por lo general tienen acceso más cierto y expedito al presupuesto nacional y a los recursos del Estado, incluyendo partidas para el nombramiento de personal. Por ejemplo, para asegurar la aprobación de la propuesta oficial para la ampliación del Canal de Panamá, en junio de 2006 el gobierno asignó a cada diputado una partida de US\$30.000 para el nombramiento de colaboradores (Alemán, 2006). A pesar de las muchas dudas y cuestionamientos razonables acerca de la viabilidad de la propuesta, el proyecto recibió la aprobación unánime de la cámara ${ }^{20}$. Mayores recursos para la designación de personal les permiten a los diputados nombrar a más simpatizantes a la burocracia de la Asamblea, lo que a su vez contribuye a fortalecer sus organizaciones de campaña y promover sus reputaciones como agentes de empleo en sus circuitos.

\section{LA ATRIBUCIÓN DE CRÉDITO Y LAS “PARTIDAS CIRCUITALES”}

Las Ilamadas "partidas circuitales", asignaciones presupuestarias a diputados, también ayudaban a los miembros de la Asamblea a robustecer su reputación en las comunidades como agentes de servicios clientelistas. Estas partidas fueron por primera vez incluidas en el presupuesto nacional en $1966^{21}$. Tras la creación de la Asamblea Legislativa en 1984 (luego de 16 años de gobierno militar), estas partidas fueron reintroducidas en 1985. Suspendidas en 1990, fueron restauradas en 1993 (un año “pre electoral”). Estuvieron disponibles a los diputados hasta el año 2000.

El propósito de estas partidas circuitales era el de permitirles a los diputados proveer "soluciones" directamente a sus electores y aumentar la visibilidad de los miembros de la Asamblea como agentes efectivos en sus comunidades. Las erogaciones bajo el programa de partidas circuitales aumentaron significativamente hasta que, por reiteradas acusaciones de mala administración y corrupción, el programa fue suspendido en 2000. Durante el último período presidencial del régimen militar (1984-1989), las erogaciones ascendieron a US $\$ 17,4$ millones. Bajo la administración del Presidente Endara, que puso las partidas circuitales a disposición de los diputados en 1993 y 1994, las erogaciones ascendieron a US\$74,1 millones. Un aumento drástico, a US\$170 millones, ocurrió bajo la administración del Presidente Ernesto Pérez Balladares (1994-1999). Esa enorme fuente de recursos atrajo a la coalición gubernamental a una cómoda mayoría de integrantes de la cámara, lo que permitió la aplicación de la agenda gubernamental sin mayores contratiempos y dio a los diputados abundantes fondos para cultivar clientelas electorales, a las que luego pudieron recurrir para solicitar y obtener votos selectivos en las elecciones de 1999 (Cochez, 2000).

La tasa de reelección ascendió de 22\% en 1989 a 26\% en 1994 y 49\% en 1999. En 2004 descendió levemente a 47\% (Guevara Mann, 2001 y cómputos del autor basados en las listas de candidatos y estadísticas electorales del Tribunal Electoral).

20 El 22 de octubre de 2006, en un referéndum nacional en el que participó tan solo el $43 \%$ del electorado, la propuesta de ampliación del Canal fue aprobada por el 78\%de los electores (Tribunal Electoral, 2006b).

21 Para una descripción detallada de las partidas circuitales y sus efectos, ver Guevara Mann, 2001. 
Los dineros canalizados a través de las partidas circuitales eran utilizados principalmente en dos actividades: mejora de infraestructura y "apoyos sociales". Las actividades de mejora de infraestructura incluían la construcción o reparación de caminos, puentes, escuelas, centros de salud, viviendas, iglesias, mercados, pistas de aterrizaje, muelles, acueductos, plantas eléctricas, alcantarillados, mataderos y otras obras públicas. Entre los "apoyos sociales" figuraban la distribución de alimentos, útiles escolares y juguetes; la compra de vehículos (tales como buses, autos para la policía, camiones y ambulancias) para uso comunitario; el financiamiento de programas nutricionales y de control de adicción, de cooperativas y de trabajos de agrimensura; la organización de salas de maternidad y de atención a adultos mayores, y la asistencia en casos de desastre, entre otros.

Los diputados se atribuían crédito por la realización de todas estas obras, a través de publicidad en los medios de comunicación mediante notas de prensa o anuncios pagados, o la colocación de letreros muy visibles indicando su "patrocinio" de dichos proyectos. Con frecuencia, hacian referencia a las ayudas sociales financiadas por las partidas circuitales como "donaciones" de los miembros de la Asamblea. El nombre del diputado frecuentemente figuraba en uniformes deportivos o vehículos adquiridos a través de su partida. Un diputado, Edgardo ("Galo") Álvarez (1994-1999), impulsó la construcción de un complejo habitacional que fue denominado "Villa Galo" en su honor.

\section{LA ATRIBUCIÓN DE CRÉDITO Y EL VOTO SELECTIVO}

Las "soluciones" comunitarias y los auxilios personales que los diputados pueden atribuirse promueven el reconocimiento del nombre del candidato entre los electores, capturan la atención de los votantes en tiempo de elecciones y facilitan el voto personalista tanto en los circuitos uninominales como en las circunscripciones plurinominales. En sus campañas, la mayoría de los candidatos a diputado en circuitos plurinominales pide el voto selectivo (Antinori, 1995: 719-20). Los candidatos que presentan antecedentes creíbles como agentes efectivos en la solución de problemas personales o comunitarios tienen mayores probabilidades de recibir el voto preferencial $-\mathrm{y}$, consecuentemente, de ser elegidos- que aquellos que no han logrado sólidas reputaciones como proveedores de bienes y servicios.

El acceso a los recursos del Estado facilita la reelección de los diputados. Pero el camino a la Asamblea no está completamente vedado a quienes, siendo ajenos a la cámara, poseen reputaciones como proveedores de servicios. El ejercicio de otros cargos públicos, como representante de corregimiento, alcalde, gobernador o empleado gubernamental, puede también sustentar la solicitud del voto selectivo, especialmente si el cargo ejercido tiene acceso a recursos presupuestarios y la comunidad considera que el funcionario "resuelve", como se dice corrientemente en Panamá.

Algunos candidatos provenientes del sector privado también pueden rodearse de una aureola de popularidad, particularmente si tienen acceso a fondos personales o familiares. El ex diputado Carlos Iván Zúñiga (1964-1968) recuerda una conversación sobre estrategias de campaña que sostuvo a finales de la década de 1980 con el miembro de una familia acaudalada que aspiraba a la diputación por un circuito integrado mayoritariamente por miembros de la clase popular. Cuando 
Zúñiga observó que el aspirante carecía de conexiones obvias con el circuito de su predilección, el candidato replicó que en Panamá la popularidad era un atributo que con recursos adecuados podía erigirse rápidamente y procedió a revelarle su plan electoral. Gracias a su conexión con una de las principales cervecerías del país, el candidato instaló varios puestos donde distribuía cerveza gratuitamente todos los viernes. Además, cada fin de mes los activistas de su campaña recogían y pagaban las cuentas de luz de varios cientos de electores. Estas tácticas rindieron buenos resultados, como lo demuestra la elección del aspirante, como diputado opositor, en 1989, sobre la base de su "servicio comunitario". Como diputado del gobierno, el personaje señalado fue reelegido en 1994 (Zúñiga, 2006).

\section{PERSONALISMO, CLIENTELISMO Y EL SISTEMA ELECTORAL}

Este trabajo ha demostrado lo siguiente:

1. A pesar de la garantía constitucional del "principio de representación proporcional", el sistema de elección de diputados panameños es, predominantemente, un sistema de mayoría relativa, con elementos de representación proporcional bajo la modalidad de lista abierta.

2. Según la teoría sobre los efectos de los sistemas electorales, ambas fórmulas (mayoría relativa y representación proporcional con lista abierta) fomentan las candidaturas personalistas.

3. Un vistazo general a las campañas para diputado en Panamá corrobora que los candidatos buscan el voto preferencial selectivo, emitido sobre bases personalistas, mucho más que el voto partidario, ideológico o programático.

4. Aun cuando el personalismo no puede atribuirse exclusivamente al sistema electoral, queda claro que éste, en su condición de institución fundamental del régimen político, suministra incentivos importantes que, en Panamá, contribuyen a estimular la búsqueda del voto selectivo.

El modelo electoral panameño opera en un ambiente político poco programático y altamente clientelista. De acuerdo con un reciente informe del Banco Interamericano de Desarrollo (BID) sobre políticas públicas en América, la política panameña es la menos programática del hemisferio. "Sobre una escala de cero (la peor nota) a ocho (la mejor), los partidos políticos panameños reciben cero en contenido programático", escribió Betty Brannan Jaén, la corresponsal de La Prensa en Washington que reseñó el informe para el diario panameño. "Esto quiere decir que Panamá es un país en que los partidos operan bajo una visión clientelista, donde los votantes juzgan al partido no por su habilidad en fomentar el bien común sino por su habilidad de distribuir beneficios a sus adeptos" (Brannan Jaén, 2006).

Aunque el objetivo de este trabajo no es explicar el contexto de clientelismo imperante en Panamá, por la íntima relación entre ese fenómeno y el personalismo, así como por el efecto negativo del clientelismo sobre el sistema democrático, vale la pena dejar anotadas algunas observaciones sobre el particular. El componente clientelista de la política panameña tiene antigua prosapia. Biesanz y Biesanz (1955) lo recalcan en un estudio sociológico publicado a mediados del siglo pasado. Janson Pérez (1993), Ricord (1989) y (en una reciente conversación con el autor) la escritora, novelista y académica Gloria Guardia destacan la merma de contenido ideológica y el predominio del clientelismo como un fenómeno que comienza a acentuarse en el primer tercio del siglo XX, luego de la 
fundación de la República en 1903 y la "modernización" del istmo²2. Ricord, por ejemplo, atribuye el menoscabo ideológico a la adopción de patrones de consumo difundidos como resultado de la vinculación más estrecha del istmo a la economía mundial, generada a partir de la construcción del canal interoceánico, inaugurado en 1914. "En su gran mayoría”, señala:

Nuestra juventud no se siente impelida por adhesiones ideológicas de ninguna clase, ni se aglutina ni empeña en luchas cívicas de aliento nacional... La degeneración de los partidos históricos en el poder, tanto liberales como conservadores, ha ofrecido el amplio cauce de esta ausencia notoria de ideales en nuestra juventud (Ricord, 1989: 114).

Para dar sustancia a sus argumentos, Ricord trae a colación el punto de vista de Guillermo Andreve, intelectual y estadista de principios del siglo XX, quien lamenta la pérdida de un estilo político más pragmático -“en que todavía se rendía culto a las ideas y en que las contiendas políticas no eran, como hoy, asunto netamente personalista, sino torneo entre partidos doctrinarios organizados a base de principios"- vigente, de acuerdo con Andreve, durante el período de unión a Colombia (1821-1903) (Ricord, 1989: 115).

A esta tradición de clientelismo recurrió el régimen militar para crearse una base de apoyo a través de la distribución de bienes y servicios públicos según criterios particularistas. El clientelismo militar, que superó con creces las prácticas del período anterior (1903-1968), fue posible gracias a la abundancia de financiamientos y donaciones extranjeras. El impulso adicional recibido durante el régimen castrense incrustó la política clientelista en todos los niveles del Estado panameño (Guevara Mann, 2001: 55-61; Linares Franco, 2006).

Para explicar la prevalencia del clientelismo pueden considerarse, además, razones socioeconómicas. En un estudio sobre la política brasileña, Ames (1995) ha alegado que donde la demanda de bienes públicos es intensa, relativamente estable y particular a ciertos sectores, los candidatos se esfuerzan por establecer reputaciones personales como proveedores de recursos a individuos o comunidades. En tales ambientes, la erección de perfiles específicos basados en la entrega de bienes públicos a las comunidades constituye un medio conveniente, aunque costoso, para conseguir el triunfo electoral (Carey and Shugart, 1995; Mainwaring, 1999: 187-90). Esa explicación, podría argumentarse, tiene validez para Panamá, cuyo índice de pobreza, incluido en el Informe de Desarrollo Humano (PNUD, 2005), fue de 37,3\% y cuyo coeficiente de Gini (desigualdad), contenido en el mismo Informe, fue de $56,4 \%{ }^{23}$.

Por lo señalado, no es lógico atribuir el clientelismo panameño únicamente a un sistema electoral que, sin duda, fomenta el voto personalista. Queda claro que el fenómeno tiene raíces culturales

22 La mercantilización del sistema político y su débil contenido ideológico hacia mediados del siglo XX son temas de trasfondo en la más reciente novela de Gloria Guardia, Lobos al anochecer (Santafé de Bogotá: Alfaguara, 2006).

23 La estadística de pobreza corresponde al período 1990-2002 y el coeficiente de Gini, al año 2002. Con respecto al coeficiente de Gini, un valor de 0 representa la igualdad perfecta y un valor de 100, la desigualdad perfecta. De los 124 países cuyo coeficiente de Gini está incluido en el Informe de Desarrollo Humano 2005, 110 reflejan coeficientes inferiores al de Panamá (o sea, tienen menos desigualdad) y trece poseen coeficientes superiores, lo cual sitúa a Panamá como un país de alta desigualdad en la distribución del ingreso (o consumo). Los países con coeficientes superiores son Zimbabwe $(56,8)$, Chile $(57,1)$, Colombia $(57,6)$, Paraguay $(57,8)$, Sudáfrica $(57,8)$, Brasil $(59,3)$, Guatemala (59,9), Swazilandia (60,9), República Centroafricana (61,3), Sierra Leona $(62,9)$, Botswana $(63,0)$, Lesotho $(63,2)$ y Namibia $(70,7)$ (PNUD, 2005). 
y socioeconómicas. Lo que resulta indiscutible es que, al privilegiar el personalismo, el sistema electoral contribuye a promover y perpetuar el clientelismo, cuyos efectos sobre la democracia no son positivos. El clientelismo, que consiste en la asignación de bienes públicos como si fueran favores personales a cambio de apoyo político (Mainwaring, 1999: 180), viola los principios de igualdad y universalidad que son cimiento del régimen democrático. Quebranta también el principio de representación democrática, que consiste en una relación pública entre el representante y los electores, en el interés colectivo de estos últimos, dirigida a promover alguna versión generalmente aceptada del bien común (Pitkin, 1967; O’Donnell, 1996). Vulnera, además, la libertad del representante, pues la abundancia de financiamiento requerida por la política clientelista y la escasez y concentración de los recursos destinables a tal fin impulsan a muchos candidatos a recurrir a patrocinadores, entre ellos el gobierno de turno, con el propósito de conseguir los fondos necesarios para obtener los votos selectivos que garanticen su elección. Esta conducta tiene consecuencias para el adecuado desempeño de las funciones legislativas y fiscalizadoras de los diputados en un régimen democrático.

Un sistema de distribución de escaños que privilegia el papel del candidato sobre el del partido contribuye a reforzar el carácter clientelista y poco programático de la política panameña. Como el voto selectivo, emitido a la par que el voto por el partido, determina la identidad de los candidatos vencedores, los aspirantes a un escaño en la Asamblea buscan activamente el voto preferencial. Para maximizar el voto selectivo, los candidatos se esfuerzan por aumentar su visibilidad y el reconocimiento de su nombre entre los electores. En la medida de sus posibilidades, ponen en funcionamiento costosas campañas publicitarias y estrategias de atribución de crédito que buscan impulsar sus reputaciones personales como proveedores eficientes de bienes y servicios a individuos y comunidades. Por todo lo anterior, a fin de salvaguardar el régimen democrático e impulsar un mejoramiento en la calidad de la democracia, convendría considerar el diseño y aplicación de un sistema electoral más proporcional y menos personalista en Panamá.

\section{REFERENCIAS}

Alemán, José Miguel. 2006. Entrevista personal, 17 de agosto.

Ames, Barry. 1995. “Electoral Strategy under Open-List Proportional Representation”. American Journal of Political Science 39 (2): 406-33.

Antinori Bolaños, Italo Isaac. 1995. "La representación política en Panamá: partidos políticos y sistema electoral". Tesis de doctorado, inédita, Universidad Complutense de Madrid. Disponible en: http: //www.ucm.es/BUCM/ tesis/19911996/S/0/S0018201.pdf.

Arias de Para, Raúl. 1984. Así fue el fraude. Panamá: Edición del autor.

Arias Zubieta, Juan. 2006. Entrevista personal, 30 de agosto.

Biesanz, John y Mavis Hiltunen Biesanz. 1955. The People of Panama. Nueva York: Columbia University Press.

Brannan Jaén, Betty. 2006. "Panamá tiene débiles políticas públicas". La Prensa, 18 de febrero. Disponible en: http: //biblioteca.prensa.com/contenido/2006/02/18/18-3a-nota1.html

Carey, John M. y Matthew Soberg Shugart. 1995. “Incentives to Cultivate a Personal Vote: A Rank Ordering of Electoral Formulas". Electoral Studies 14 (4): 417-439.

Cochez, Guillermo. 2000. “El cinismo del ex ministro Jorge Eduardo Ritter”. El Universal, 15 de junio.

Guardia, Gloria. 2006. Entrevista personal, 15 de agosto.

Guevara Mann, Carlos. 2001. "Forsaken Virtue: An Analysis of the Political Behavior of Panamanian Legislators, 19841999". Tesis de doctorado (PhD), inédita, Universidad de Notre Dame. 
Guevara Mann, Carlos. 2004. "Calidad de la representación política y tamaño de las circunscripciones electorales: una comparación de las asambleas panameñas de 1945 y 1999”. Revista de Ciencia Política 24 (2): 94-115.

Janson Pérez, Brittmarie. 1993. "The Process of Political Protest in Panama, 1968-1989". Tesis de doctorado (PhD), inédita, Universidad de Texas en Austin (Publicada en castellano como En nuestras voces: Panamá protesta, 19681989. Panamá: Editorial La Prensa, 1993).

Katz, Richard. 1980. A Theory of Parties and Electoral Systems. Baltimore: Johns Hopkins University Press.

Koster, Richard M. y Guillermo Sánchez Borbón. 1990. In the Time of the Tyrants: Panama, 1968-1990. New York: W.W. Norton.

Linares Franco, Julio. 2006. “Lobos al anochecer”. El Panamá América, 30 de junio. Disponible el 26 de octubre de 2006 en http://www.pa-digital.com.pa/archive/06302006/index.htm.

Mainwaring, Scott. 1999. Rethinking Party Systems in the Third Wave of Democratization: The Case of Brazil. Stanford: Stanford University Press.

Mayhew, David. 1974. Congress: The Electoral Connection. New Haven and London: Yale University Press.

Morice, Eugenio. 2000. “Polito, no esperes justicia”. La Prensa, 31 de marzo.

Nohlen, Dieter. 2006. "La reforma del sistema binominal desde una perspectiva comparada". Revista de Ciencia Política 26 (1): 191-202.

O'Donnell, Guillermo. 1996. “Illusions About Consolidation”. Journal of Democracy 7 (2): 34-51.

Pitkin, Hanna Fenichel. 1967. The Concept of Representation. Berkeley: University of California Press.

Programa de las Naciones Unidas para el Desarrollo (PNUD). 2005. Informe de Desarrollo Humano 2005. Nueva York: PNUD. Disponible en: http://hdr.undp.org/reports/global/2005/espanol/pdf/HDR05_sp_complete.pdf

Putnam, Robert. 1993. Making Democracy Work: Civic Traditions in Modern Italy. Princeton: Princeton University Press.

Quintero, César. 1967. Derecho constitucional. Vol. I. San José: A. Lehmann.

República de Panamá. 1994. Constitución política de la República de Panamá de 1972, reformada por los actos reformatorios de 1978, por el acto constitucional de 1983 y los actos legislativos 1 de 1993 y 2 de 1994. Disponible el 8 de octubre de 2006 en: http://www.binal.ac.pa/buscar/clconst.htm.

República de Panamá. 2003. Código Electoral. Panamá: Tribunal Electoral. Disponible el 8 de octubre de 2006 en: http:// www.tribunal-electoral.gob.pa/elecciones/docum_electoral/documentos/codigo-electoral-2003.pdf.

República de Panamá. 2004. Constitución política de la República de Panamá de 1972, reformada por los actos reformatorios de 1978, por el acto constitucional de 1983 y los actos legislativos 1 de 1993, 2 de 1994 y el acto legislativo 1 de 2004. Disponible el 8 de octubre de 2006 en: http://www.binal.ac.pa/buscar/clconst.htm.

Reynolds, Andrew y Ben Reilly. 1997. The International IDEA Handbook of Electoral System Design. Estocolmo: Instituto Internacional para la Democracia y la Asistencia Electoral (International IDEA).

Ricord, Humberto E. 1989. Panamá en la guerra de los mil días. Panamá: Edición del autor, con la colaboración del Instituto Nacional de Cultura (INAC) y la cooperación del Ministerio de Educación.

Rosas, Jorge Rubén. 2006. Entrevista personal, 16 de agosto.

Sánchez, Salvador. 2006. Comunicación por correo electrónico, 6 de octubre.

Tejada Mora, Juan Antonio. 2006. Entrevista personal, 28 de agosto.

Tribunal Electoral de Panamá. 1999a. "Elecciones: Documentos de interés electoral”. Disponible el 8 de octubre de 2006 en: http://www.tribunal-electoral.gob.pa/elecciones/docum_electoral/elec_celebradas.html.

Tribunal Electoral de Panamá. 1999b. “Elecciones: Elecciones 1999-Resultados”. Disponible el 8 de octubre de 2006 en: http://www.tribunal-electoral.gob.pa/elecciones/elecciones1999/cuadros/elec-01.html

Tribunal Electoral de Panamá. 2004a. "Distribución de curules legislativas por partido y tipo de proclamación: Elecciones populares del 2 de mayo de 2004". Disponible el 6 de octubre de 2006 en: http://www.tribunal-electoral.gob. pa/elecciones/elecciones-2004/doc/cuadro-07.xls.

Tribunal Electoral de Panamá. 2004b. “Elecciones 2004". Disponible el 8 de octubre de 2006 en: http://www.tribunalelectoral.gob.pa/elecciones/elecciones-2004/proyeccion.html.

Tribunal Electoral de Panamá. 2004c. "Votos obtenidos por candidatos a legisladores en la República por partido político y alianza, según circuito electoral: Elecciones populares del 2 de mayo de 2004”. Disponible el 8 de octubre de 2006 en: http://www.tribunal-electoral.gob.pa/elecciones/elecciones-2004/doc/cuadro-05.xls.

Tribunal Electoral de Panamá. 2006a. "Propuesta de circuitos". Disponible el 8 de octubre de 2006 en: http://www. tribunal-electoral.gob.pa/proyecto/propuesta-circuitos.html

Tribunal Electoral de Panamá. 2006b. “Referéndum 2006”. Disponible el 26 de octubre de 2006 en: http://201.227.244.69/ referendum2006/webappelections/.

Tribunal Electoral de Panamá, IV Comisión Nacional de Reformas Electorales. 2006. "Proyecto de Ley por la cual se reforma el Código Electoral”. Disponible el 8 de octubre de 2006 en: http://www.tribunal-electoral.gob.pa/ proyecto/proyectodeley.html. 
Valdés Escoffery, Eduardo. 2002. "Evolución de los sistemas electorales aplicados a la elección de legisladores en la República de Panamá". Trabajo presentado en el Primer Curso Internacional de Derecho Parlamentario, Panamá, 22-26 de julio. Disponible en: http://www.tribunal-electoral.gob.pa/publicaciones/publicaciones/documentos/folleto-magvaldez.pdf

Zúñiga, Carlos Iván. 2006. Entrevista personal, 1 de agosto.

Carlos Guevara Mann es Profesor Asistente de Ciencia Política y Director del Programa de Asuntos Internacionales en la Universidad de Nevada-Reno. Recibió el Doctorado (PhD) en la Universidad de Notre Dame, Indiana, Estados Unidos. Consultor de organismos internacionales y columnista del diario La Prensa, fue Director General de Política Exterior de la Cancillería panameña. Es autor de Panamanian Militarism: A Historical Interpretation (Ohio University Press, 1996), publicada en castellano bajo el título llegitimidad y hegemonía: Una interpretación histórica del militarismo panameño (Editorial La Prensa, 1994). Sus campos principales de investigación y publicación son la democracia y la democratización, la representación política y las relaciones entre Estados Unidos y América Latina.

(E-mail: guevara@unr.edu) 